\title{
Identification of Prognostic Genes Associated with Expression Differentiation During Metabolism Process in Hepatocellular Carcinoma (HCC)
}

\section{Xin Yang}

The first affiliated hospital of university of south china

\section{Chuang-jie Cao}

the first affiliated hospital of university of south china

Cheng-Yun Dou

the first affiliated hospital of university of south china

Hai-Bo Tang

the first affiliated hospital of university of south china

Qiong Liu

the first affiliated hospital of university of south china

Juan Zou

university of south china

\section{Yu-kun Li}

University of South China https://orcid.org/0000-0002-5169-4693

\section{Tian Zeng}

university of south china

Xia Xie ( $\nabla$ xiexia1109@sina.com )

First Affiliated Hospital of University of South China

\section{Primary research}

Keywords: Bioinformatical analysis, hepatocellular carcinoma, metabolic-genomic landscape, the cancer genome atlas, prognostic index

Posted Date: October 8th, 2020

DOI: https://doi.org/10.21203/rs.3.rs-86583/v1

License: (c) (1) This work is licensed under a Creative Commons Attribution 4.0 International License.

Read Full License 


\section{Abstract}

Background: Metabolic disorders have attracted more and more attention from scientists in the research of various tumors, especially in hepatocellular carcinoma (HCC). The purpose of this study is to assess the prognostic significance of metabolism in HCC.

Methods: The expression profile of metabolism-related gene (MRGs) were extracted from the cancer genome atlas (TCGA) database of 349 HCC-surviving patients. Subsequently, A series of biomedical computational algorithms were used to identify seven-MRGs signature as a prognostic model. GSEA analysis indicated the function and pathway enrichment of these MRGs. Then, drug sensitivity analysis found the hub gene, which tested by IHC staining.

Results: 420 differential MRGs and 116 differential transcription factor (TFs) expression were extracted from HCC patients based on TCGA database. Metabolic disturbance might be involved in the development of HCC by GO and KEGG enrichment analyses. LASSO regression analysis constructed seven-MRGs signature (DHDH, EN01, G6PD, LPCAT1, PDE6D, PIGU and PPAT), which were used to predict the prognosis of HCC patients. Further GSEA analysis found the function and pathway enrichment of these seven MRGs. Then, drug sensitivity analysis indicated G6PD might play a key role in the prognosis of HCC by chemoresistance. Finally, we used IHC staining to demonstrate the relationship of G6PD expression and clinical parameter in HCC patients.

Conclusion: This study provides a potential clue for predicting the prognosis of HCC patients and further studies on HCC metabolism.

\section{Background}

Hepatocellular carcinoma (HCC) ranks sixth in incidence (more than 800 thousand new cases) and fourth in overall mortality (more than 780 thousand deaths), which means it is responsible for about 1 in 10 cancer cases and deaths in 2018[1]. According to the examination of histopathological classification, $\mathrm{HCC}$ is one of the main causes of liver cancer, accounting for more than 80 percent[2]. Surgical resection of liver cancer and complete and delicate liver transplantation are effective methods for the treatment of $\mathrm{HCC}$. However, the poor clinical outcomes of HCC is still leaded by many causes, such as local recurrence, chemotherapeutic resistance, and distant metastasis[3]. Moreover, due to the occult onset of HCC and the lack of specific early markers, most patients with HCC are often diagnosed at the advanced stage with local or distant metastases, and their average survival time is approximately 6 months[4]. Therefore, the identification of specific early diagnostic markers for $\mathrm{HCC}$ and the resolution of local recurrence, chemotherapeutic resistance and distant metastasis are urgent problems to be solved in clinical practice. The occurrence and development of HCC is an extremely complex pathological process, and its molecular mechanism remains inenarrable[5]. A great volume of studies has led to a consistent found that various cancer microenvironmental factors, including high pro-inflammatory cytokines induce by oxidation and endoplasmic reticulum stress, hyperinsulinemia, changes in the intestinal microbiome, and adipokines 
dysregulation, are thought to coordinate the progression of $\mathrm{HCC}[6-11]$. But the most important risk factor for HCC is actually represented by excess body fat[12]. Although the mechanisms of excess body fat promoting carcinogenesis are still not fully understood in HCC, several studies have shown that cancer cell-intrinsic metabolic reprogramming play a significant role in promoting carcinogenesis by obesity.

Metabolic reprogramming can promote carcinogenesis by disturbing signaling pathways, inducing cell adaptation, regulating cellular differentiation, and modulating epigenetic states[13]. Changes in glucose metabolism are one of the markers of tumor metabolism. The most typical example is the Warburg effect which induce aerobic glycolysis instead of mitochondrial oxidative phosphorylation to promote cancer cell proliferation[14]. Changes in lipid metabolism are also a marker of cancer metabolism. fatty acids functions include signaling molecules, energy, structural components of cell membranes, and storage compounds, which are necessary for cancer cell proliferation. However, circulating exogenous lipids are preferentially used by normal cells, while cancer cells, such as liver cancer cells, perform higher rates of de novo lipids[15]. Moreover, another well-known metabolic disorder is increased glutaminolysis to make more citrate and a-ketoglutarate, which can enhance the activation of the mitochondrial tricarboxylic acid (TCA) cycle.

Cancer metabolic reprogramming is a complex molecular network, which are increasingly got attention. The difference between cancer and normal cell metabolic reprogramming is helpful for more accurate diagnosis and prognosis evaluation of HCC patients[16]. At present, bioinformatics analysis is a powerful tool in deriving information about HCC[17]. Our aim in this study is to get several metabolism-related genes (MRGs) identified as potential candidates for targeted HCC treatment. We assessed the level and prognosis of MRGs, and made an individualized prognosis for HCC patients. Bioinformatics analysis was performed to explore the potential regulatory mechanisms. The results of this study can provide a basis for further in-depth metabolism-related work and provide a good prospect for individualized treatment of HCC.

\section{Materials And Methods}

\section{The source data and preprocessing of Metabolism-related genes (MRGs) and transcription factors}

70 MRGs sets were downloaded from the KEGG database of GSEA website, and a total of 1466 MRGs were included, with the same method as above[18]. 318 tumor related transcription factors is extracted from cistrome database (http://cistrome.org/), to assist the integrated analysis of the molecular mechanisms of cancer development.

\section{TCGA data acquisition}

Our study included information from the TCGA database on a total of 349 HCC patients who were followed up for at least one month, with survival time ranging from 30 days to 3675 days. Download level 
3 FPKM data for subsequent analysis. Univariate Cox regression was used to analyze mRNAs related to patient survival time for model construction.

\section{Model construction and prognosis}

Patients with HCC were randomly divided into training set and test set (7:3) in TCGA. Firstly, the univariate cox risk proportional regression model was selected in the training set to initially screen the genes related to prognosis $(p<0.05 \&(H R>1.5$ or $H R<0.5))$, and lasso regression was used to further construct the prognostic correlation model. After the expression values of each specific gene were included, the risk score formula for each patient was constructed and weighted by its estimated regression coefficients in the lasso regression analysis. According to the risk score formula, patients were divided into low risk group and high risk group according to the score value. Survival differences between the two groups were assessed by kaplan-meier and compared using log-rank statistical methods. Lasso regression analysis and stratified analysis were used to examine the role of risk scores in predicting patient outcomes.ROC curve was used to study the accuracy of model prediction.

\section{Gene ontology (GO) and Kyoto Encyclopedia of Gene and Genome (KEGG) analyses}

GO, an important bioinformatics analysis, contains biological processes (BPs), cellular component (CC), and molecular function (MF). KEGG is a complex analysis database resource of gene function system, which connects genomic information with higher-order functional information. David is used for GO and KEGG analysis. The cut-off standard was $p<0.05$.

\section{The analysis of GSEA}

GSEA analysis uses predefined gene sets, sorts genes according to the degree of differential expression in the two types of samples, and then checks whether the preset gene sets are enriched at the top or bottom of the list.In this study, GSEA was used to compare the differences in signaling pathways between the high-risk group and the low-risk group to explore the possible molecular mechanism of the difference in prognosis between the two groups, in which the replacement times were set as 1000 and the replacement type as phenotype.

\section{Hub gene analysis}

GSCALite (http://bioinfo.life.hust.edu.cn/web/GSCALite/) is a cancer genomic analysis platform that integrates cancer genomics data from TCGA for 33 cancer types, drug response data from GDSC and CTRP, and normal tissue data from GTEx for genomic analysis in a one-to-one data analysis process. In this study, GSCALite was used for pathway analysis of key hub genes, GDSC and CTRP drug sensitivity analysis[19].

\section{Patients and specimens}

We collected paraffin-embedded tissue blocks, which included $10 \mathrm{HCC}$ tissues and corresponding paracarcinoma tissues from the Department of Pathology, The First Affiliated Hospital of University of South 
China. 30 Tissue sections from all HCC cases were reviewed by a pathologist. All HCC patients were classified according to the 7th Union for International Cancer Control TNM staging system. The clinical data of the specimens recorded in detail are accurate and complete. All HCC patients were regularly followed up for 4 to 81 months to assess postoperative survival. The collection and use of tissues followed the procedures according to the ethical standards as formulated in the Helsinki Declaration. Written informed consent was obtained from each patient, which was approved by the research ethics committee of the University of South China.

\section{Immunohistochemistry (IHC) staining}

For IHC staining, these slices were dewaxed by xylene. Subsequently, them were rehydrated with a graded ethanol series, repaired antigen with microwave. Endogenous peroxidase activity were blocked by endogenous peroxidase for $20 \mathrm{~min}$ at $37^{\circ} \mathrm{C}$. After rinsing, these slides were incubated for overnight at $4^{\circ} \mathrm{C}$ with a first antibody that was diluted with PBST in a working solution (1: 1000 dilution, Ab231828, Abcam, MA, USA). After rinsing, subsequent operations follow this paper[20]. The IHC score refer to this previous paper[21].

\section{Statistical analysis}

All statistical analyses were conducted in the R language (version 3.6). All the statistical tests were bilateral, and $p<0.05$ was statistically significant.

\section{Results}

\section{Identification the survival-associated MRGs in HCC}

A growing body of studies indicated a significant role of cancer metabolic reprogramming in HCC occurrence, development and progression. In order to ascertain MRGs about HCC prognosis, the analysis of differential gene expression was utilized. Firstly, the expression profiles of MRGs were extracted from TCGA HCC data set, which contained 1466 MRGs (Fig. 1A). Subsequently, we found that 357 differentially expressed genes (DEGs) was up-regulated, and 63 DEGs was down-regulated $(p<0.05$ \& $\log |\mathrm{FC}|>1$ ) in these MRGs between HCC and normal liver samples via Volcano Plot (Fig. 1B).

\section{Functional enrichment was determined by GO and KEGG analysis}

These key MRGs were mapped utilized GO terms and KEGG analysis, which suggested relevant functions and underlying mechanisms in HCC progression. Furthermore, we found that the MRGs were largely correlated with biological process (BP) of metabolism progression, including small molecule catabolic process, phospholipid metabolic process and organic hydroxy compound metabolic process. In the part of cellular component (CC) and molecular function (MF), them were associated with mitochondrial matrix and cofactor binding, respectively (Fig. 2A). For the analysis of KEGG, purine metabolism, drug metabolism-other enzymes and oxidative phosphorylation were the most frequent enrichment (Fig. 2B). 


\section{Validation the differentially expressed transcription factors in HCC}

Previous research has illustrated how relationships of transcription factors (TFs) are highly related with the expression of MRGs. Similar to the study of Kalliopi Makarona[22], the expression of glucose 6phosphate dehydrogenase (G6PD) could be enhanced by the HDAC inhibitor. Therefore, we firstly extracted the data of transcription factors in HCC and normal liver samples, which contain $318 \mathrm{TFs}$ (Fig. 3A). Furthermore, 202 TF had not changed significantly, 8 TFs were upregulated, and 108 TFs were downregulated ( $p<0.05 \& \log |\mathrm{FC}|>1$ ) (Fig. 3B). In the analysis of GO, we found this set of TFs enriched in $\mathrm{BP}, \mathrm{CC}$ and MF terms, including covalent chromatin modification, chromatin, and chromatin binding, respectively (Fig. 3 C). In the analysis of KEGG, this set of TFs involved in cell cycle, cellular senescence, and hepatocellular carcinoma (Fig. 3D). The functional enrichment analysis indicated these TFs might be involved in the development of HCC. Moreover, The PPI network also found extensive interactions between differentially expressed MRGs and key TFs, consistent with previous reports that MRGs and TFs in metabolic reprogramming worked together to form a delicate process conducive to cancer progression. After disconnected nodes deleted, the PPI network was shown in Fig. 3E. The regulatory network based on TFs clearly illuminated the regulation relationship among these MRGs

\section{Construction and validation of MRGs Signature}

For constructing signature associated with MRGs, 32 MRGs were inputted to least absolute shrinkage and selection operator (LASSO) regression. Then, the seven hub MRGs (DHDH, EN01, G6PD, LPCAT1, PDE6D, PIGU, PPAT) were inputted to the LASSO regression to construct a prognostic signature which divided HCC patients into two groups, and the two groups had discrete clinical outcomes by the seven hub MRGs (Fig. 4A). The prognostic model was as follows:

$[\mathrm{Y}=\mathrm{DHDH} * 0.658+\mathrm{ENO} 1 * 0.052+\mathrm{G} 6 \mathrm{PD} * 0.043+\mathrm{LPCAT} 1 * 0.147+\mathrm{PDE} 6 \mathrm{D} *(-0.154)+\mathrm{PIGU} 0.040+\mathrm{PPAT} *$ 0.604].

According to the optimal cut off point of 1.471, the risk score of these HCC patients can be divided into low and high-risk groups (Fig. 4B). The survival score and survival status of HCC patients was visualized by Fig. 5A and B. The high expression of DHDH, EN01, G6PD, LPCAT1, PIGU and PPAT, and the low expression of PDE6D were found in HCC patients with higher risk scores (Fig. 4D). Nevertheless, survival time and rates decreased with increased risk scores (Fig. 4B and C).

The application of ROC curve and KM plot were utilized to evaluate the role of this prognostic model in predicting the prognosis of HCC patients. In this dataset, the overall survival (OS) of high-risk group is higher than low-risk group, which has significant difference according statistic analysis $(P<0.05)$

(Fig. 5A). Furthermore, the area under curve of the receiver operating characteristic (ROC) of 12,36 and 60 month were $0.778,0.728$ and 0.749 , respectively (Fig. 5B). Moreover, univariate and multivariate Cox regression analysis was used to further evaluate the performance of our model in patients with HCC by using other common prognostic factors. In univariate Cox regression model, tumor stage, $T$ and seven- 
gene signature could be utilized as independent prognostic factor for survival (Fig. 5C). In multivariate Cox regression, only risk score had prognostic effects (Fig. 5D).

\section{Validation of the prognostic marker of the seven-mRNA signature in HCC}

To verify the prognostic effect of the prognostic model on HCC, we used test set of HCC patients in TCGA to measure the prognostic effect of the model. The results indicated that the test set of HCC patients were classified into high-risk and low-risk group by risk score and median cut-off point (Fig. 6A). The survival time of high-risk group was significantly lower than low-risk group (Fig. 6B). The area under curve of the ROC of 12,36 and 60 month were $0.796,0.744$ and 0.675 , respectively (Fig. $6 \mathrm{C}$ ). Taken together, these results of test set were consistent with those of the training set, which indicated the seven-mRNA signature could anticipate the prognosis of HCC (between Figs. 5 and 6).

\section{GSEA analysis of MRGs signature}

In order to investigate the potential mechanism of seven-gene signature on prognosis, we utilized GSEA enrichment analysis. In GO terms, GSEA using the gene sets indicated the enrichment in categories like monocarboxylic acid catabolic process, organic acid catabolic process, and positive regulation of cell cycle phase transition (Fig. 7A). In KEGG terms, GSEA analysis suggested the enrichment in categories, including cell cycle, complement and coagulation cascades, and fatty acid metabolism (Fig. 7B).

\section{Verification of the drug sensitivity of hub MRGs}

GSCALite (http://bioinfo.life.hust.edu.cn/web/GSCALite/) is a cancer genome analysis platform based on web[19]. We utilized the database to make the drug sensitivity analysis of seven hub MRGs, offering help for drug choice for these hub MRGs targeted treatment. Therefore, we found that drug sensitivity of the two hub MRGs (PPAT and G6PD) were obviously associated with multiple chemotherapy drugs by utilizing the GSCALite database, and the results were exhibited in Supplementary Figure S1-2, offering help for drug treatment by targeting hub MRGs. Furthermore, we also found that the pathophysiological function of G6PD was enriched in apoptosis and cell cycle by pathway analysis based on GSCALite ( Supplementary Figure S3).

\section{G6PD expression is increased and correlated with clinical parameters in HCC}

To further confirm the expression level of G6PD in HCC, we detected the level of G6PD by IHC. As we expected in the above results, the expression of G6PD is enhanced in cancer tissues of 10 patients with HCC compared to para-carcinoma tissues(Fig. 8). The results indicated that G6PD might promote the development and progression of HCC.

\section{Discussion}


Along with the rapid development of high-throughput technologies, DNA chips and second-generation sequencing technologies have generated massive amounts of data, and researchers need bioinformatics to process useful information[23]. Bioinformatics is an interdisciplinary subject combining computer and life science, which comprehensively computer science, statistics, biological science and other theories to calculate and analyze the potential significance of extensive biological data[24]. More and more bioinformatics platforms and analysis software have come into being. Their data contains genome information and functional integration, which can significantly improve the extraction and analysis of biological data. Data sharing based on platforms also effectively reduces the experimental and time cost.

Changes in cancer metabolic processes such as glucose metabolism and amino acid metabolism are characteristic of cancer[25]. The characteristics of metabolomics can better understand the pathophysiological changes of $\mathrm{HCC}$ and provide great potential for the development of new methods for HCC treatment[26]. For instance, These metabolic pathways (bile-acid biosynthesis, tryptophan metabolism, urea-cycle metabolism, and Citric acid cycle) were significantly changed in HCC group[27]. Previous study indicated that glycolysis and amino acid metabolism had a closely association with the development and progression of HCC by multi-omics analyses[28, 29]. Moreover, abnormal lipid metabolism has also been found in HCC patients[30].

In this study, we studied MRGs and TFs changes in HCC patients by bioinformatics. After identifying $X$ MRGs in the TCGA data sets as capable of identifying key clinicopathological features of HCC, we established risk signatures of MRGs by LASSO regression analysis. We identified the seven-gene prognostic model of $\mathrm{HCC}$ by using the formula to calculate the prognostic score: $\left[\mathrm{Y}=\mathrm{DHDH}{ }^{\star} 0.658+\right.$ $\mathrm{ENO} 1 * 0.052+\mathrm{G} 6 \mathrm{PD} * 0.043+\mathrm{LPCAT} 1 * 0.147+\mathrm{PDE} 6 \mathrm{D} *(-0.154)+\mathrm{PIGU} * 0.040+\mathrm{PPAT} * 0.604]$. According to our risk signature, patients in the high-risk group tend to be associated with poor prognosis, which has a significantly higher expression of DHDH, EN01, G6PD, LPCAT1, PIGU, PPAT, but lower expression of PDE6D.

The model signature genes EN01, G6PD, LPCAT1, PIGU, and PDE6D have been found to be involved in the development and progression of HCC. ENO1 (a-enolase), a key enzyme of glycolysis, can promote the conversion of 2-phosphoglycerate to phosphoenolpyruvate 1, which can enhance the ability of proliferation in $\mathrm{HCC}[31]$. Furthermore, previous studies have shown that ENO1 is upregulated in HCC tissues, which is related to tumor differentiation and progression[32]. G6PD (Glucose-6-phosphate dehydrogenase) is the first enzyme and rate limiting enzyme of pentose phosphate pathway[33]. The expression of G6PD in HCC patients and HCC cell lines are increased, which can promote migration and invasion by epithelial-mesenchymal transition (EMT)[34]. LPCAT1 (lysophosphatidylcholine acyltransferases 1) can acylate the unsaturated acyl group to maintain the integrity of the cell membrane[35], which can increase the ability of cell proliferation, migration and invasion in HCC[36]. PIGU (Phosphatidylinositol glycan anchor biosynthesis class $U$ ) plays its carcinogenic role by enhancing GPI-T activity and anchor-binding substrates including urokinase plasminogen activator surface receptors[37], which relate to poor prognosis in HCC, and nomogram-based risk scores that combine PIGU level with the standard TNM tend to be a more powerful set of vehicle for predicting prognosis[38]. 
Finally, Peter Dietrich and his colleagues found that PDE6D (rod-specific photoreceptor cGMP phosphodiesterase) might affect different cytoplasmic and nuclear pathways in HCC, as well as other types of cancer. PDE6D could promote the ability of proliferation, migration, invasion and sorafenib resistance in HCC cells, so PDE6D's great potential might be a new therapeutic and diagnostic target for HCC progression and chemotherapy resistance.

To investigate the potential molecular mechanisms by which gene signatures influence prognosis, GSEA analysis was utilized. The results showed that the gene expression changes in the prognosis model mainly affected monocarboxylic acid catabolic process, organic acid catabolic process, and positive regulation of cell cycle phase transition, providing clues for further research. In KEGG terms of GSEA, cell cycle was the most affected pathophysiological pathway. In summary, these seven hub signatures gene might influence cell cycle to mediate the HCC progression by multiple metabolic pathways. Furthermore, the drug sensitivity analysis based on GSCALite indicated that G6PD might be involved in the resistance of multiple chemotherapy drugs, contributed by apoptosis and cell cycle. However, the relationship between HCC progression and G6PD expression still be not confirmed, and our correlation analysis between IHC staining and clinical pathological parameters might provide some clues to research in this area. Taken together, our result suggested that MRGs, especially in G6PD, might play a role in the development and progression of HCC.

\section{Conclusion}

We used HCC metabolome and TFs data to identify the differentially expressed MRGs. we constructed a seven-gene signature and verified that this signature was used to predict the prognosis of patients with HCC. Furthermore, we detected the expression of G6PD that is a key role in the seven-gene signature, and analyzed its correlation with clinical parameters. Further study of these MRGs will provide new insights into the potential relationship between metabolic disorders and HCC progression.

\section{Declarations}

\section{Ethics approval and consent to participate}

The collection and use of tissues followed the procedures according to the ethical standards as formulated in the Helsinki Declaration. Written informed consent was obtained from each patient, which was approved by the research ethics committee of the University of South China.

\section{Consent for publication}

Written informed consent for publication was obtained from all participants.

\section{Availability of data and materials}


The data used to support the findings of this study are available from the corresponding author upon request.

\section{Competing interests}

The authors have no conflicts of interest.

\section{Funding}

This study is supported by Hengyang City 2018 guidance Project (S2018F9031021273).

\section{Authors' contributions}

Conception and design: Xin Yang and Xia Xie. Collection and assembly of data: Chuang-jie Cao and Cheng-Yun Dou. Data analysis and interpretation: Hai-Bo Tang and Qiong Liu. Manuscript writing: Xin Yang. Paper revision: Xia Xie. Final approval of manuscript: All authors.

\section{Acknowledgements}

The authors would like to thank the DAVID, GTEX and TCGA databases for the availability of the data.

\section{References}

1. Bray, F., et al., Global cancer statistics 2018: GLOBOCAN estimates of incidence and mortality worldwide for 36 cancers in 185 countries. CA Cancer J Clin, 2018.

2. Akinyemiju, T., et al., The Burden of Primary Liver Cancer and Underlying Etiologies From 1990 to 2015 at the Global, Regional, and National Level: Results From the Global Burden of Disease Study 2015. JAMA Oncol, 2017. 3(12): p. 1683-1691.

3. Li, Y.K., et al., Human p21-activated kinase 5 (PAK5) expression and potential mechanisms in relevant cancers: Basic and clinical perspectives for molecular cancer therapeutics. Life Sci, 2019. 241: p. 117113.

4. Hollebecque, A., et al., Systemic treatment of advanced hepatocellular carcinoma: from disillusions to new horizons. Eur J Cancer, 2015. 51(3): p. 327-39.

5. Braicu, C., et al., Hepatocellular Carcinoma: Tumorigenesis and Prediction Markers. Gastroenterology Res, 2009. 2(4): p. 191-199.

6. Nakagawa, H., et al., ER stress cooperates with hypernutrition to trigger TNF-dependent spontaneous HCC development. Cancer Cell, 2014. 26(3): p. 331-343.

7. Park, E.J., et al., Dietary and genetic obesity promote liver inflammation and tumorigenesis by enhancing IL-6 and TNF expression. Cell, 2010. 140(2): p. 197-208.

8. Nakagawa, H., How endoplasmic reticulum stress contributes to obesity-driven hepatic tumorigenesis. Hepat Oncol, 2015. 2(3): p. 209-211. 
9. Yoshimoto, S., et al., Obesity-induced gut microbial metabolite promotes liver cancer through senescence secretome. Nature, 2013. 499(7456): p. 97-101.

10. Arano, T., et al., Serum level of adiponectin and the risk of liver cancer development in chronic hepatitis C patients. Int J Cancer, 2011. 129(9): p. 2226-35.

11. Shalapour, S., et al., Inflammation-induced IgA+ cells dismantle anti-liver cancer immunity. Nature, 2017. 551(7680): p. 340-345.

12. Anstee, Q.M., et al., From NASH to HCC: current concepts and future challenges. Nat Rev Gastroenterol Hepatol, 2019. 16(7): p. 411-428.

13. Ward, P.S. and C.B. Thompson, Metabolic reprogramming: a cancer hallmark even warburg did not anticipate. Cancer Cell, 2012. 21(3): p. 297-308.

14. Vander, H.M., L.C. Cantley and C.B. Thompson, Understanding the Warburg effect: the metabolic requirements of cell proliferation. Science, 2009. 324(5930): p. 1029-33.

15. Budhu, A., et al., Integrated Metabolite and Gene Expression Profiles Identify Lipid Biomarkers Associated With Progression of Hepatocellular Carcinoma and Patient Outcomes. Gastroenterology, 2013. 144(5): p. 1066-1075.e1.

16. Tian, Y., et al., ER-residential Nogo-B accelerates NAFLD-associated HCC mediated by metabolic reprogramming of oxLDL lipophagy. Nat Commun, 2019. 10(1): p. 3391.

17. Zhang, Q., et al., Landscape and Dynamics of Single Immune Cells in Hepatocellular Carcinoma. Cell, 2019. 179(4): p. 829-845.e20.

18. Lin, P., et al., Development of a prognostic index based on an immunogenomic landscape analysis of papillary thyroid cancer. Aging (Albany NY), 2019. 11(2): p. 480-500.

19. Liu, C.J., et al., GSCALite: a web server for gene set cancer analysis. Bioinformatics, 2018. 34(21): p. 3771-3772.

20. Su, B., et al., Diallyl disulfide suppresses epithelial-mesenchymal transition, invasion and proliferation by downregulation of LIMK1 in gastric cancer. Oncotarget, 2016. 7(9): p. 10498-512.

21. X, G., et al., Over-expression of TFB2M facilitates cell growth and metastasis via activating ROS-AktNF-KB signaling in hepatocellular carcinoma. Liver international : official journal of the International Association for the Study of the Liver, 2020.

22. Makarona, K., et al., Transcriptional and epigenetic basis for restoration of G6PD enzymatic activity in human G6PD-deficient cells. Blood, 2014. 124(1): p. 134.

23. Wang, M., et al., Identification of Key Genes and Prognostic Value Analysis in Hepatocellular Carcinoma by Integrated Bioinformatics Analysis. Int J Genomics, 2019. 2019: p. 3518378.

24. Do, V.I., et al., Network integration of multi-tumour omics data suggests novel targeting strategies. Nat Commun, 2018. 9(1): p. 4514.

25. Kroemer, G. and J. Pouyssegur, Tumor cell metabolism: cancer's Achilles' heel. Cancer Cell, 2008. 13(6): p. 472-82. 
26. Poto, C.D., et al., Metabolomic Characterization of Hepatocellular Carcinoma in Patients with Liver Cirrhosis for Biomarker Discovery. Cancer Epidemiol Biomarkers Prev: p. cebp;1055-9965.EPI-160366v2.

27. Liang, Q., et al., Phenotypic Characterization Analysis of Human Hepatocarcinoma by Urine Metabolomics Approach. Sci Rep, 2016. 6: p. 19763.

28. Xie, Q., et al., Multi-omics analyses reveal metabolic alterations regulated by hepatitis B virus core protein in hepatocellular carcinoma cells. Sci Rep, 2017. 7: p. 41089.

29. Li, Z.F., et al., Gas chromatography/time-of-flight mass spectrometry-based metabonomics of hepatocarcinoma in rats with lung metastasis: elucidation of the metabolic characteristics of hepatocarcinoma at formation and metastasis. Rapid Commun Mass Spectrom, 2010. 24(18): p. 2765-75.

30. Metabolomic profiles of hepatocellular carcinoma in a European prospective cohort. Bmc Medicine. 13(1): p. 242.

31. Hamaguchi, T., et al., Glycolysis module activated by hypoxia-inducible factor 1 alpha is related to the aggressive phenotype of hepatocellular carcinoma. Int J Oncol, 2008. 33(4): p. 725-31.

32. Takashima, M., et al., Overexpression of alpha enolase in hepatitis?C virus-related hepatocellular carcinoma: Association with tumor progression as determined by proteomic analysis. 2010. 5(6): $\mathrm{p}$. 1686-1692.

33. Stanton, R.C., Glucose-6-phosphate dehydrogenase, NADPH, and cell survival. lubmb Life, 2012. 64(5): p. 362-369.

34. Lu, M., et al., Elevated G6PD expression contributes to migration and invasion of hepatocellular carcinoma cells by inducing epithelial-mesenchymal transition. Acta Biochim Biophys Sin (Shanghai), 2018. 50(4): p. 370-380.

35. Agarwal, A.K., et al., Functional characterization of human 1-acylglycerol-3-phosphate-0acyltransferase isoform 9: cloning, tissue distribution, gene structure, and enzymatic activity. $J$ Endocrinol, 2007. 193(3): p. 445-57.

36. Morita, Y., et al., Lysophosphatidylcholine acyltransferase 1 altered phospholipid composition and regulated hepatoma progression. J Hepatol, 2013. 59(2): p. 292-9.

37. Guo, Z., et al., CDC91L1 (PIG-U) is a newly discovered oncogene in human bladder cancer. Nat Med, 2004. 10(4): p. 374-81.

38. Cao, J., et al., PIGU overexpression adds value to TNM staging in the prognostic stratification of patients with hepatocellular carcinoma. Hum Pathol, 2019. 83: p. 90-99.

\section{Figures}



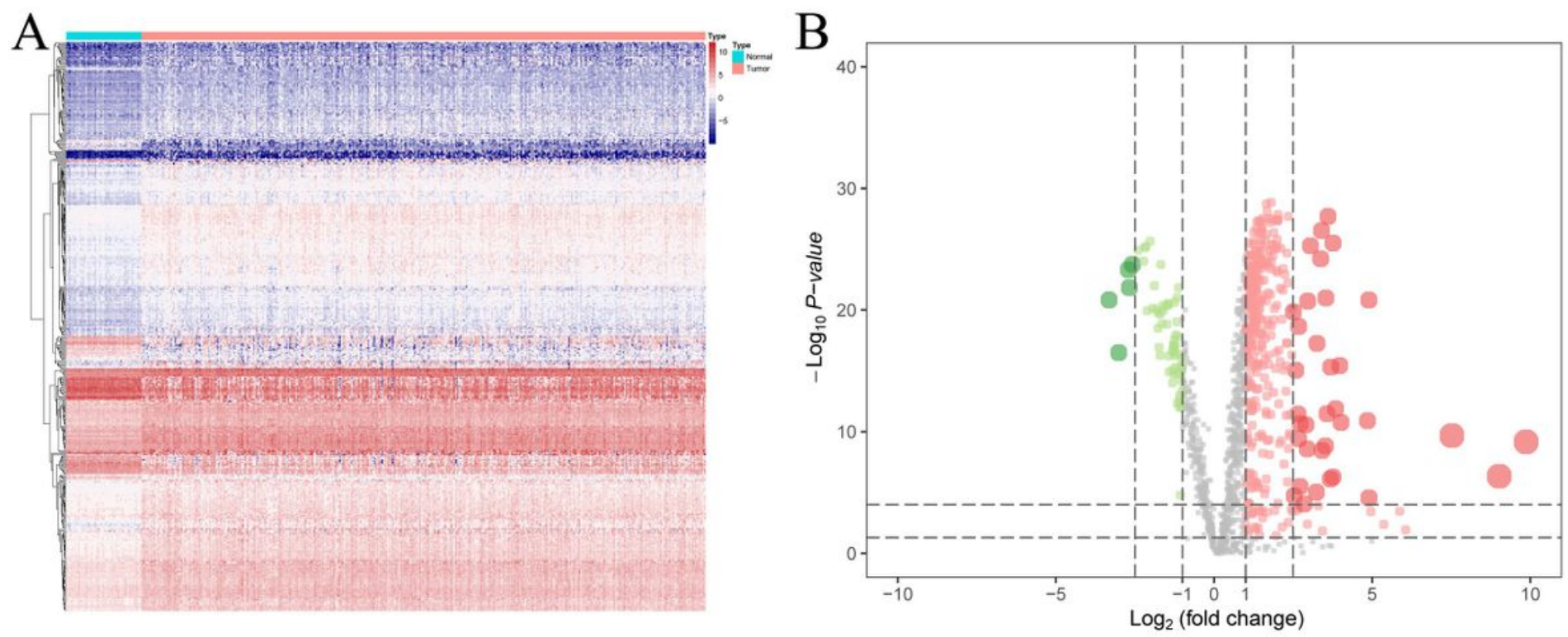

\section{Figure 1}

Identification the survival-associated MRGs in HCC (A) Heat map shows the significant MRGs in TCGA datasets. (B) Volcano plot shows 1466 MRGs in HCC. Red means up-regulation significantly. Green means down-regulation significantly. $(p<0.05 \& \log |F C|>1)$ 
A

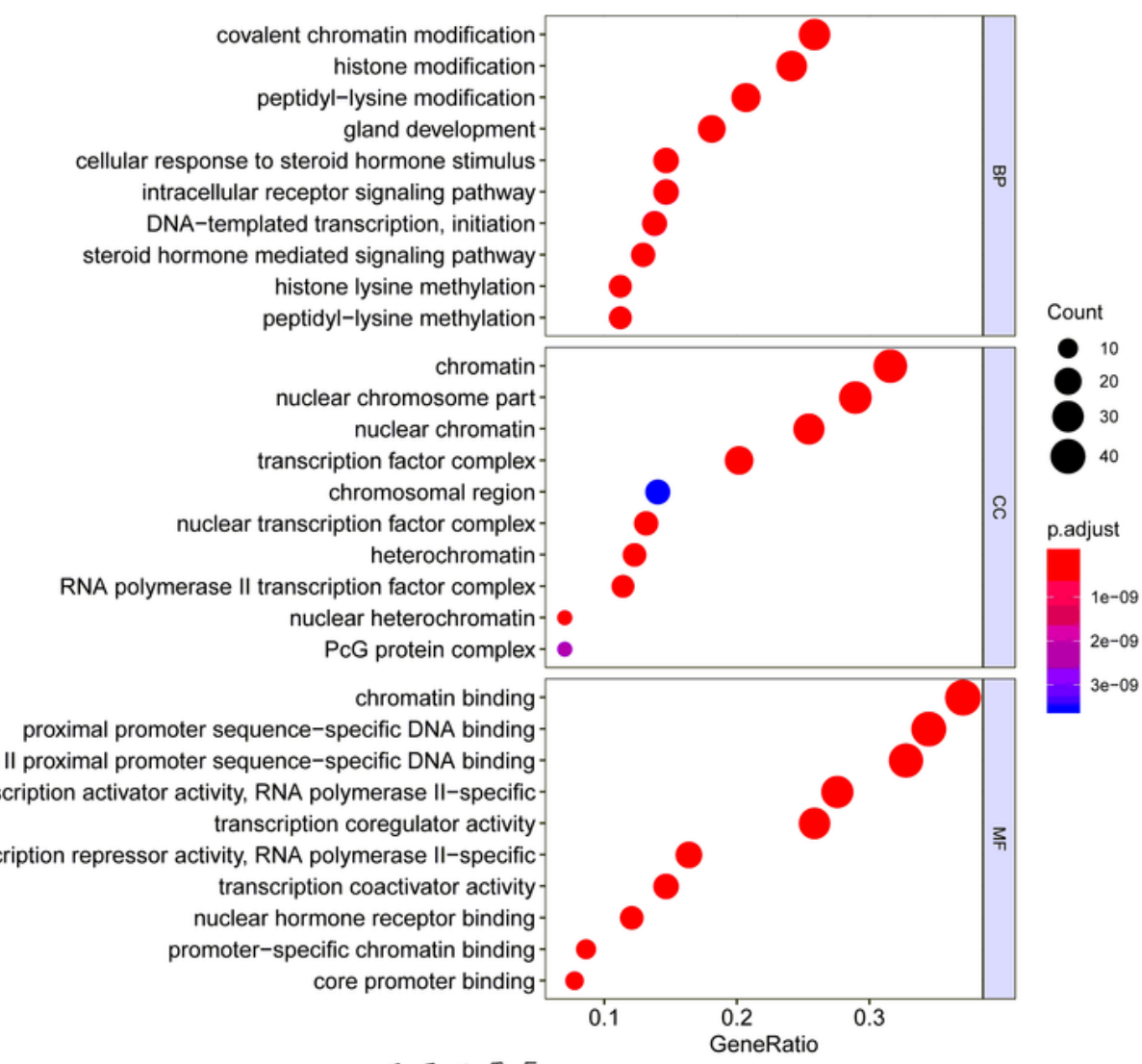

$\mathrm{B}$

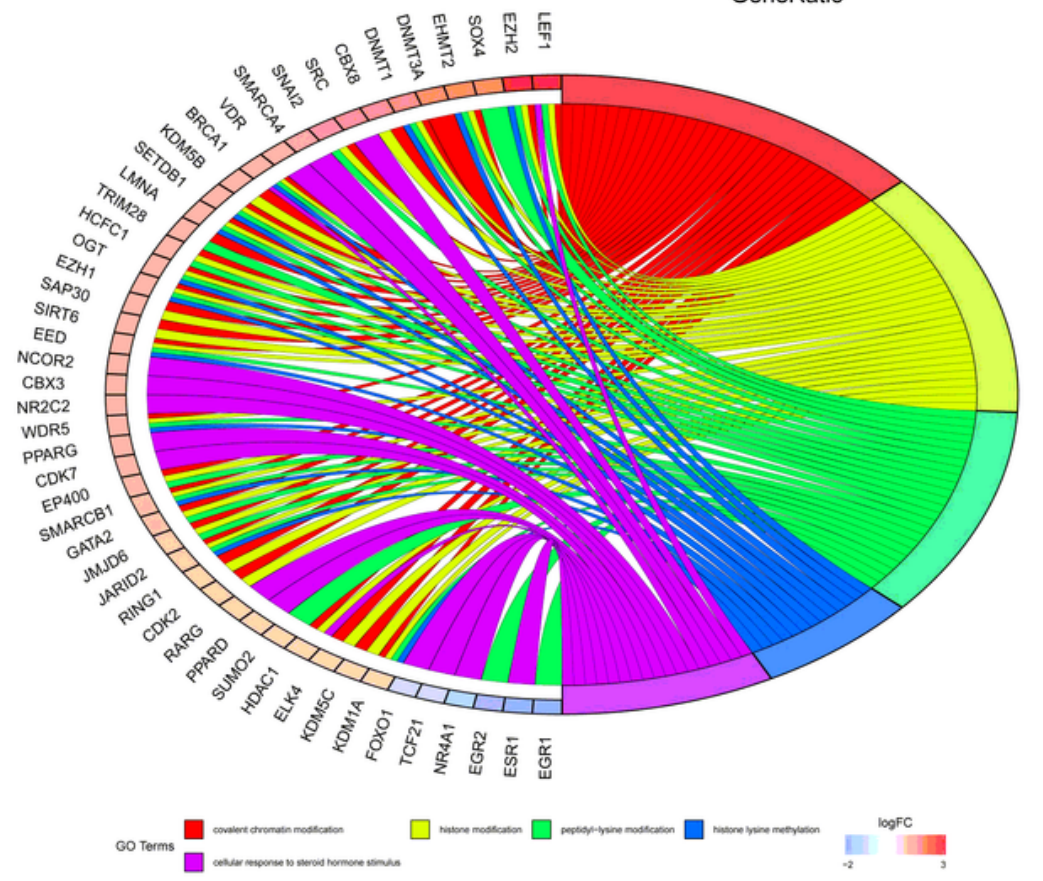

\section{Figure 2}

Gene Ontology analyze of these MRGs. (A) In the part of biological process (BP), cellular component (CC) and molecular function (MF), them were associated with small molecule catabolic process, mitochondrial matrix and cofactor binding, respectively. (B) KEGG enrichment analysis indicated that purine metabolism, drug metabolism-other enzymes and oxidative phosphorylation were the most frequent enrichment for these MRGs. 

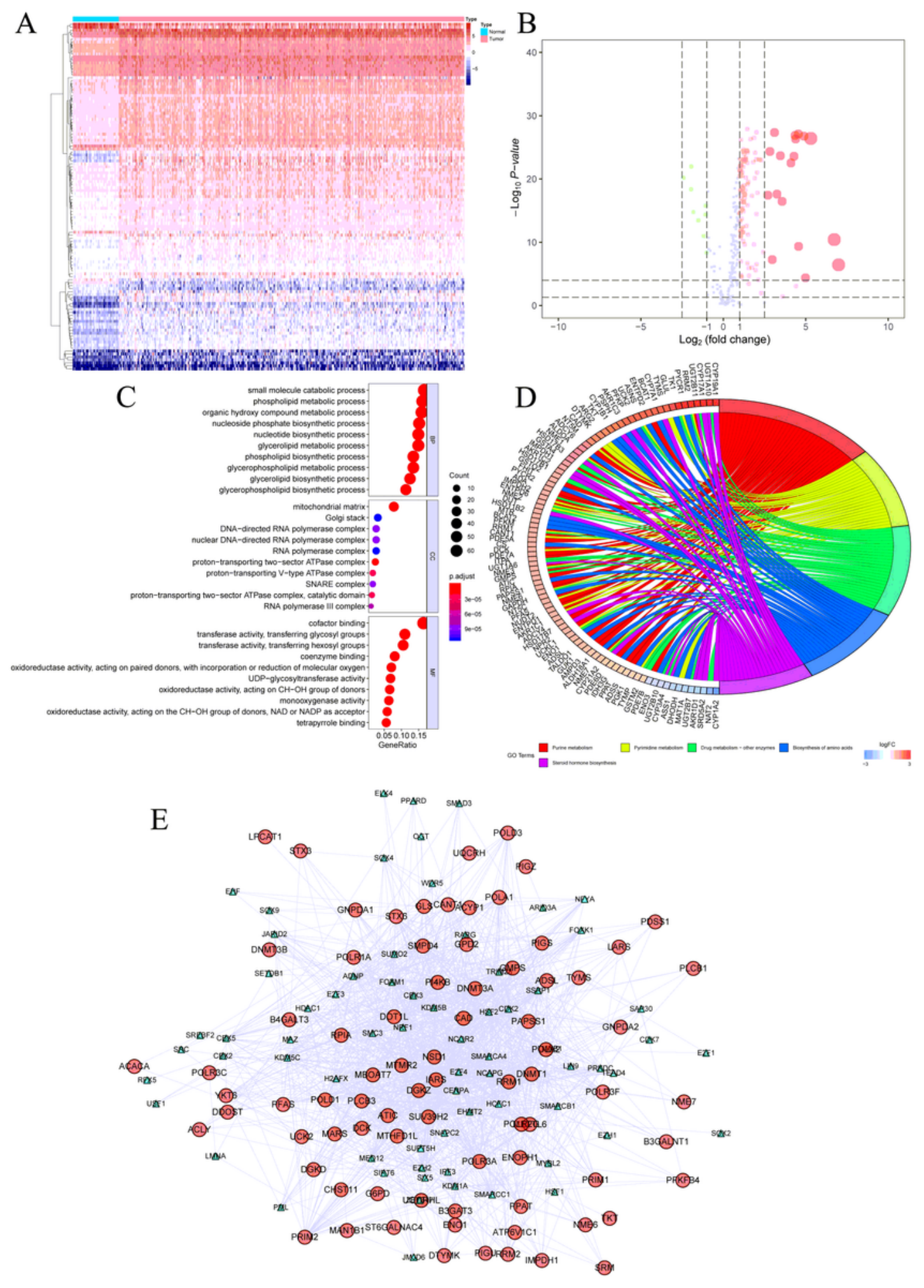

\section{Figure 3}

Validation the differentially expressed TFs in HCC (A) Heat map shows the significant HCC related TFs in TCGA datasets. (B) Volcano plot shows 318 TFs in HCC. Red means up-regulation significantly. Green means down-regulation significantly. (C) In the part of BP, CC and MF, them were associated with covalent chromatin modification, chromatin, and chromatin binding, respectively. (D) KEGG enrichment analysis indicated that cell cycle, cellular senescence, and hepatocellular carcinoma were the most frequent 
enrichment for these TFs. (E) The PPI network also found extensive interactions between differentially expressed MRGs and key TFs. $(p<0.05 \& \log |F C|>1)$
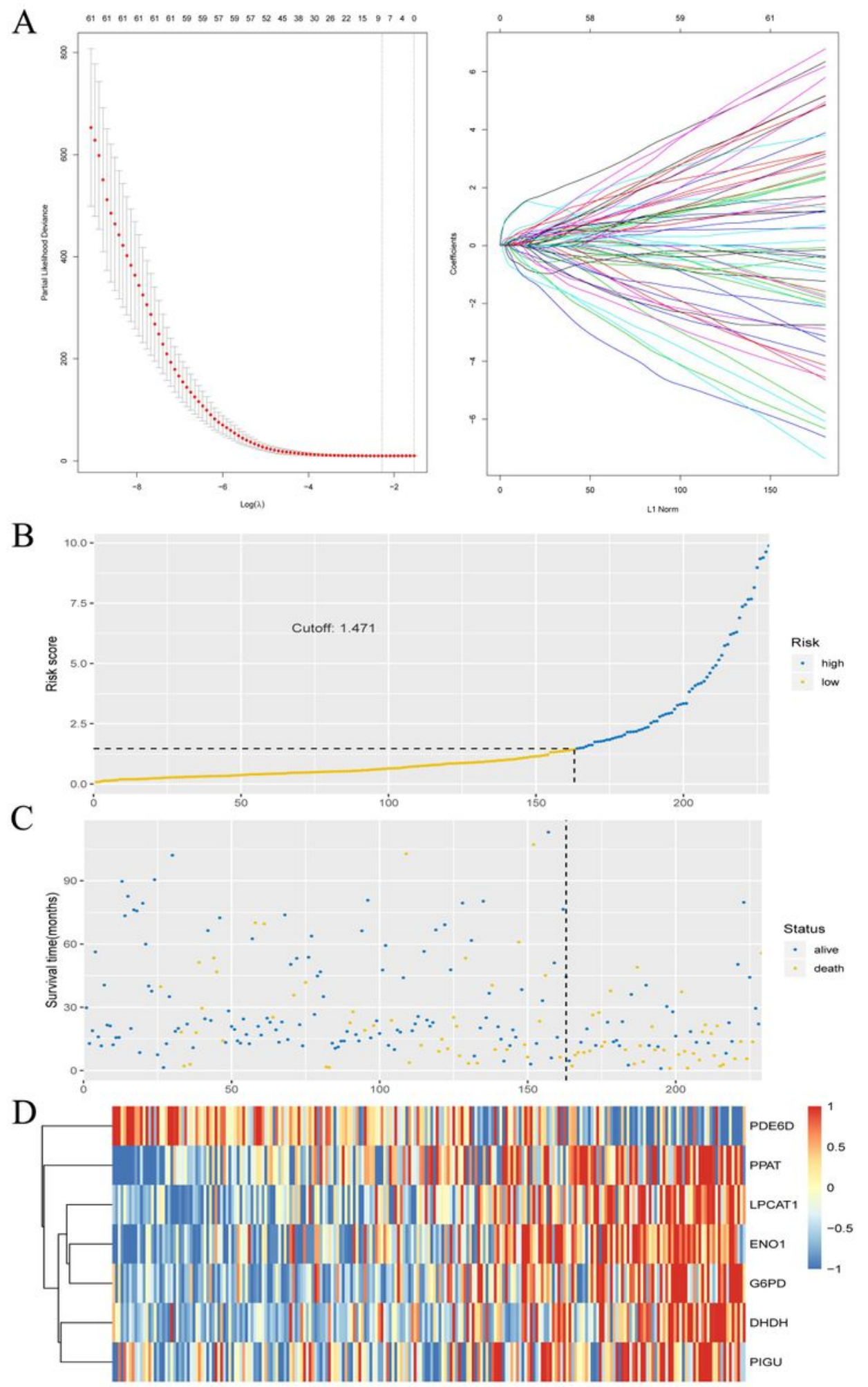

Figure 4

Construction and validation of MRGs Signature (A) Construction of prognostic signatures based on LASSO regression analysis. (B) Risk score analysis of the seven-gene signature of HCC. Risk score of gene signature. (C) Duration of cases. (D) low and high score groups for the seven genes. 

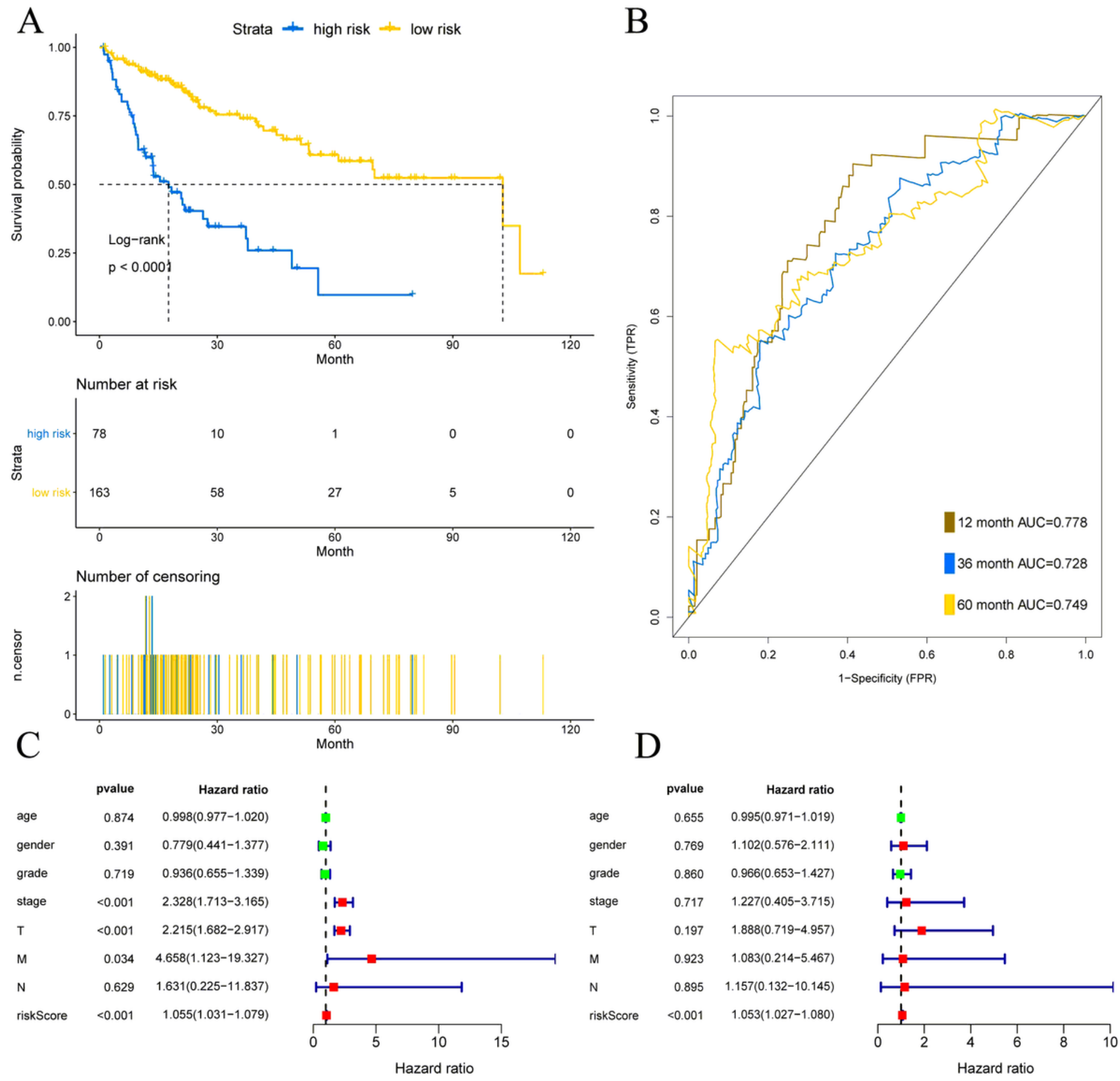

\section{Figure 5}

Seven-MRGs signature in HCC patients based on TCGA in training set (A) Survival curve of low risk and high risk groups classified by seven-MRGs signature. (B) The area under curve of ROC of low risk and high risk groups by seven-MRGs signature based on TCGA. (C) Univariate Cox regression model was used to analyze the prognostic effects of seven-MRGs signatures and common prognostic factors. (D) The prognostic effect of seven-MRGs signature and used common prognostic factors by multivariate Cox regression model. 
A
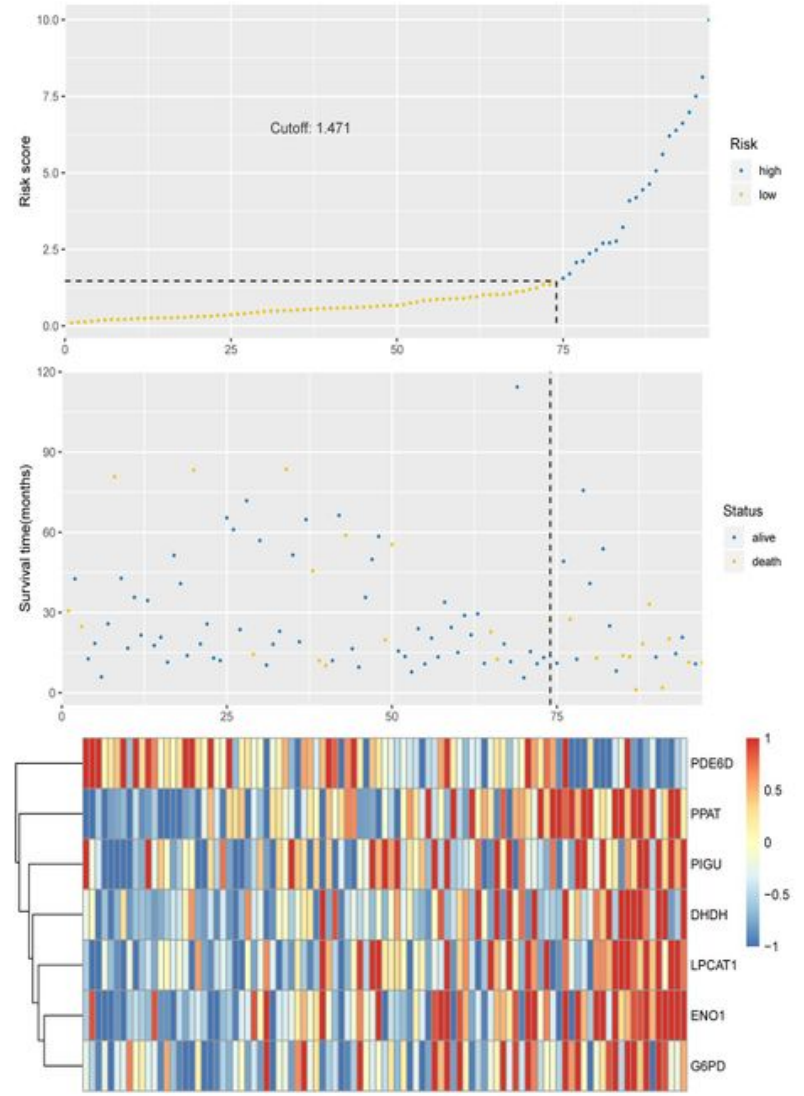

$\mathrm{C}$

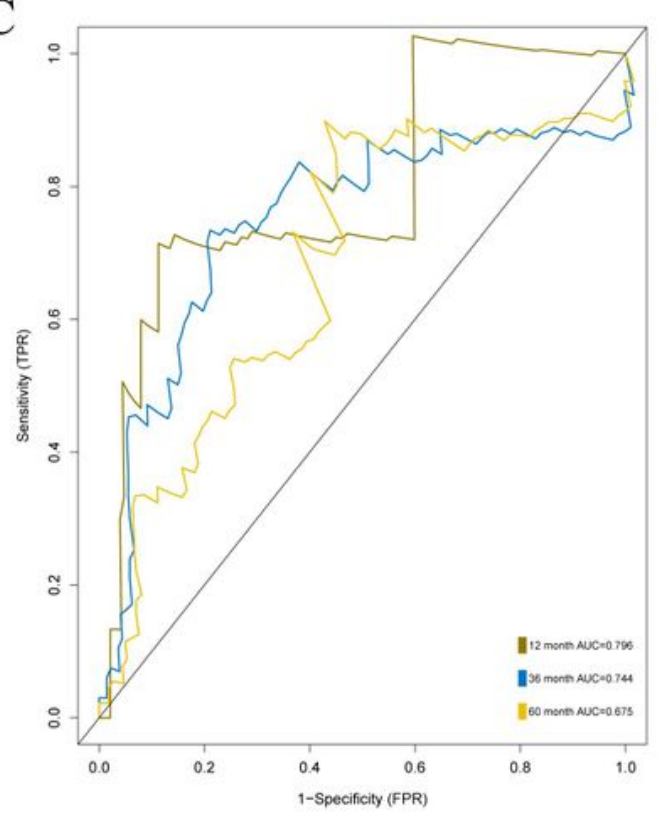

B

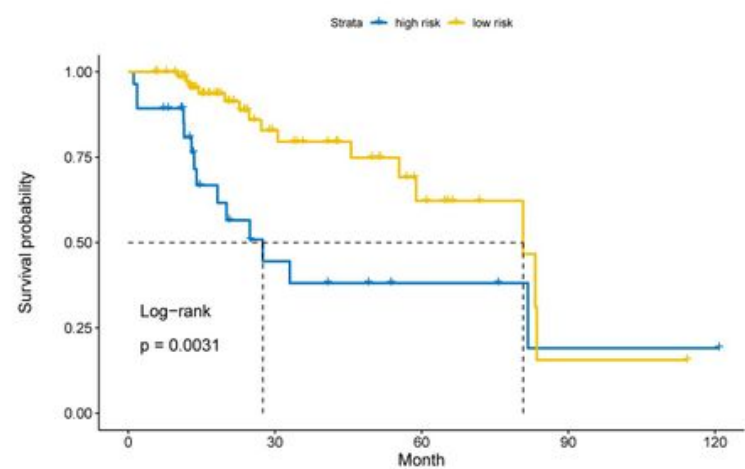

Status
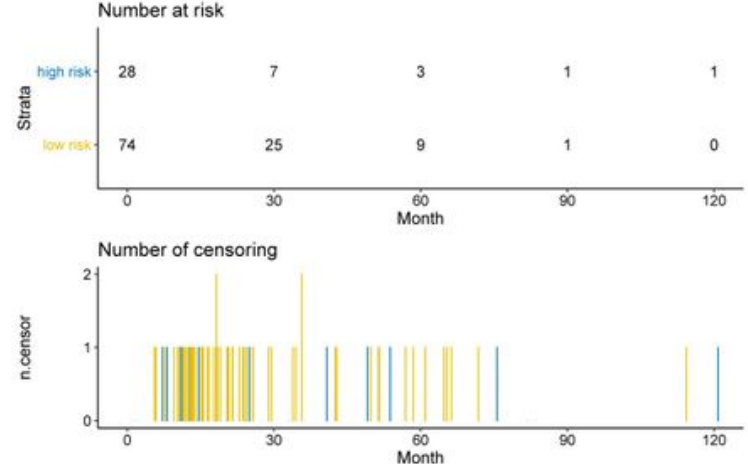

\section{Figure 6}

The prognostic capability of the seven-MRGs signature in test set. (A) Risk score analysis of the sevengene signature of HCC in test set. (B) Survival curve of low risk and high risk groups classified by sevenMRGs signature in test set. (C) The area under curve of ROC of low risk and high risk groups by sevenMRGs signature based on TCGA in test set. 

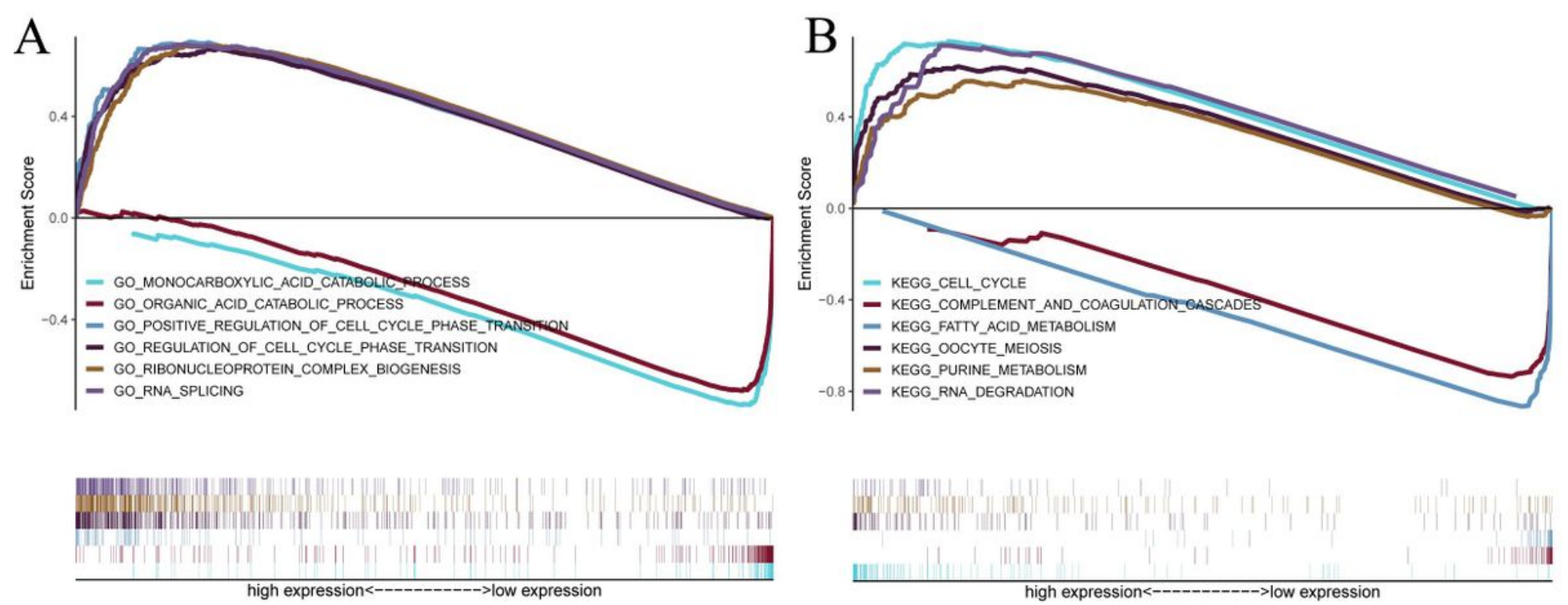

Figure 7

GSEA analysis for the seven MRGs (A) In GO terms, GSEA indicated that monocarboxylic acid catabolic process, organic acid catabolic process, and positive regulation of cell cycle phase transition were most significantly enriched. (B) In KEGG terms, GSEA suggested the enrichment in cell cycle, complement and coagulation cascades, and fatty acid metabolism.

Para-carcinoma tissue

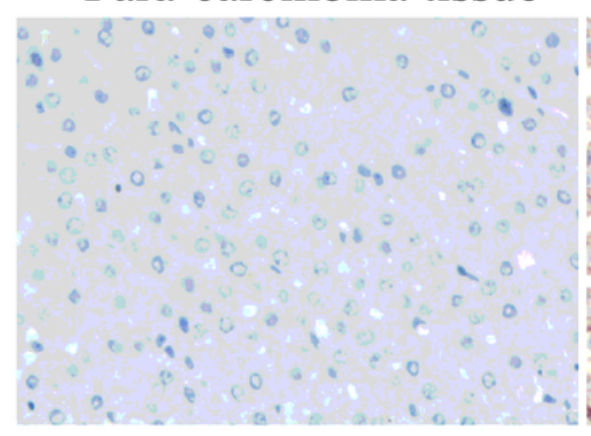

HCC tissue

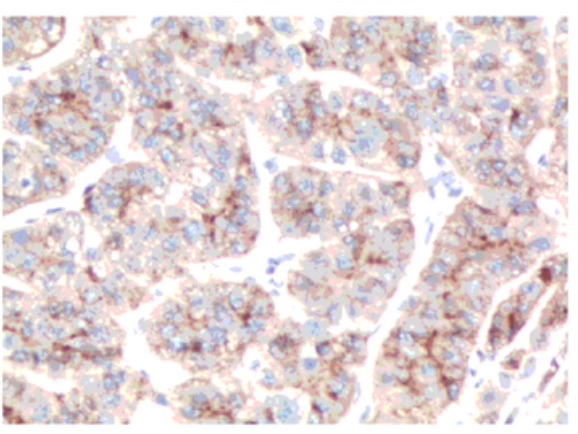

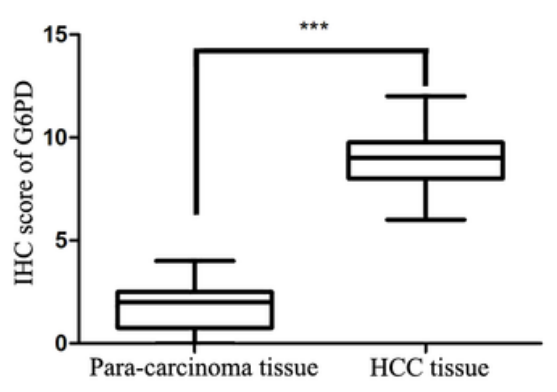

\section{Figure 8}

G6PD was over-expressed and has correlated with prognosis in HCC patients Immunohistochemical staining of G6PD expression in HCC and para-carcinoma tissue. G6PD showed strong cytoplasm expression in HCC.

\section{Supplementary Files}

This is a list of supplementary files associated with this preprint. Click to download.

- SupplementaryFigureS1.jpg 
- SupplementaryFigureS2.jpg

- SupplementaryFigureS3.jpg 